\title{
Evolution spatiale et temporelle des dégâts du jasside Jacobiella facialis Jacobi, 1912 (Cicadellidae) en comparaison avec la distribution de la pluviométrie au cours des vingt dernières années dans les zones de culture cotonnière en Côte d'Ivoire
}

\author{
Pitou Woklin Euloge KONE ${ }^{1,2^{*}}$, Germain Elisabeth Cynthia OCHOU ${ }^{1,2}$, \\ Gouzou Juste Roland DIDI ${ }^{1,2}$, Sekpa Charles DEKOULA ${ }^{3,4}$, Malanno KOUAKOU ${ }^{2}$, \\ Kouadio Kra Norbert BINI ${ }^{2}$, Dagnogo MAMADOU ${ }^{1}$ et Ochou Germain OCHOU ${ }^{2}$ \\ ${ }^{I}$ Université Nangui Abrogoua, Laboratoire de Cytologie et de Biologie Animales, \\ 02 BP 801 Abidjan 02, Côte d'Ivoire. \\ ${ }^{2}$ Centre National de Recherche Agronomique, Station de Recherche sur le Coton, \\ Laboratoire Entomologie, 01 BP 633 Bouaké 01, Côte d'Ivoire. \\ ${ }^{3}$ Centre National de Recherche Agronomique, Laboratoire Central Sols, \\ Eaux et Plantes, 01 BP 633 Bouaké 01, Côte d'Ivoire. \\ ${ }^{4}$ Université Félix Houphouët Boigny, Laboratoire de Sciences et Techniques de l'Eau et de l'Environnement, \\ 22 BP 582 Abidjan 22, Côte d'Ivoire. \\ *Auteur correspondant,E-mail : eulogepitou@gmail.com; Tel : +22509765048
}

\section{RESUME}

Le jasside Jacobiella facialis est un ravageur piqueur-suceur du cotonnier en Côte d'Ivoire. Depuis quelques années, ses attaques sont persistantes sur tout le cycle cultural malgré les traitements insecticides. Cette étude visait à analyser les distributions spatio-temporelles des dégâts du ravageur en relation avec les évolutions pluviométriques des vingt dernières années. De 1993 à 2015, des relevés parasitaires et pluviométriques ont été réalisés sur plus de 300 parcelles paysannes. Les données ont révélé une variation interannuelle des niveaux moyens de dégâts $(0,52-6,01$ plants attaqués pour 30 plants) avec une tendance à la hausse. La période 2011-2015 correspond à une forte pression parasitaire (3,05-6,01 plants attaqués pour 30 plants), avec un pic d'attaques entre le $93^{\text {ème }}$ et $114^{\text {ème }}$ jour après levée (JAL), contrairement à la période 19932007 correspondant à une faible pression parasitaire $(0,52-2,10$ plants attaqués pour 30 plants) avec un pic d'attaques entre le $30^{\text {ème }}$ et $44^{\text {ème }} \mathrm{JAL}$. La régression de la pluviométrie de juin-juillet explique de façon significative la recrudescence du ravageur. En effet, la distribution des plus fortes infestations dans le Nord-Est correspond au cours des dernières années à une pluviométrie inférieure à $320 \mathrm{~mm}$. Cette donne recommande l'élaboration d'une nouvelle stratégie de gestion efficace contre le ravageur.

(C) 2017 International Formulae Group. All rights reserved.

Mots clés : Jasside, pluviométrie, évolution spatio-temporelle, cotonnier, protection, Côte d'Ivoire. 


\title{
Spatial and temporal variation of the injury levels of jassid Jacobiella facialis Jacobi, 1912 (Cicadellidae) in relation to rainfall variation for the last two decades in the cotton growing area of Côte d'Ivoire
}

\begin{abstract}
Jassid Jacobiella facialis is a sucking pest of cotton in Côte d'Ivoire. For the last decades, its attacks are persistent throughout the entire crop cycle despite insecticide treatments. This study aimed at analyzing the spatial and temporal distributions of pest damage in relation to rainfall trends over the past two decades. From 1993 to 2015, pest and rainfall surveys were carried out through a network involving more than 300 fields. Data revealed an annual variation in the pest damage levels, fluctuating between 0.52 and 6.01 attacked plants for 30 plants, with an upward trend from 1993 to 2015. The period 2011-2015 corresponds to a high pest pressure (3.05-6.01 attacked plants for 30 plants), with a peak of attack between the $93^{\text {rd }}$ and $114^{\text {th }}$ days after emergence (DAE), while the period 1993-2007 corresponds to a low pest pressure (0.52 to 2.10 attacked plants for 30 plants) with a peak of attacks between the $30^{\text {th }}$ and $44^{\text {th }}$ DAE. The regression of the rainfall of june-july explains significantly the resurgence of the pest. In fact, the distribution of high pest pressure in the Northeast corresponds, during the last decade, particularly to rainfalls lower than $320 \mathrm{~mm}$. This suggests the development of a new strategy for effective management of $J$. facialis.

(C) 2017 International Formulae Group. All rights reserved.
\end{abstract}

Keywords: Jassid, rainfall, spatial and temporal variation, cotton, protection, Côte d'Ivoire.

\section{INTRODUCTION}

La culture cotonnière joue un rôle primordial dans l'épanouissement socioéconomique des populations en zone de savane des régions Centre et Nord de la Côte d'Ivoire. Cette source de revenu est pourtant mise à mal par un parasitisme particulièrement important et diversifié.

La réussite de la culture cotonnière nécessite non seulement un bon itinéraire technique mais aussi et surtout un bon contrôle des insectes ravageurs qui sont susceptibles de causer des pertes de récoltes variant de $50 \%$ à $75 \%$ selon les pays, les années et les localités (Badiane et al., 2015 ; Sarr et al., 2016). Pour assurer le contrôle des ravageurs, la lutte chimique demeure la méthode la plus adoptée par les producteurs.

Néanmoins, l'utilisation massive en

Côte d'Ivoire de produits insecticides, notamment des pyréthrinoïdes pour contrôler les lépidoptères du cotonnier, s'est traduite par une sélection d'individus résistants chez des ravageurs comme Helivoverpa armigera (Martin et al., 2000). Il s'est donc bâti, depuis 1998, autour de ce ravageur majeur, une stratégie de gestion et de prévention de la résistance aux pyréthrinoïdes (Ochou et Martin, 2002 ; Djihinto et al., 2016). Elle vise à réduire l'utilisation des pyréthrinoïdes en faisant appel à leurs alternatives chimiques (benzoylurées, diamides, organophosphorés, avermectines, etc.) pour assurer une partie des traitements foliaires.

Malgré l'adoption de ce programme, il est observé, depuis ces dernières années, une émergence de certains ravageurs dont le jasside Jacobiella facialis. En effet, un grand nombre de producteurs se plaignent de la persistance des attaques de cette cicadelle 
malgré les traitements insecticides. Cette situation soulève des interrogations, notamment, sur la sensibilité du jasside aux produits insecticides utilisés, sur le niveau de pilosité suffisant ou non des variétés vulgarisées, sur l'impact des traitements insecticides sur l'entomofaune utile ou encore sur les conditions climatiques et environnementales actuelles devenues propices à la pullulation des populations du ravageur.

La résurgence du jasside $J$. facialis est susceptible de remettre en cause la stratégie de protection en vigueur dont l'efficacité repose en grande partie sur une bonne maitrise de la distribution spatio-temporelle des principaux ravageurs; d'où l'importance de la surveillance parasitaire. La présente étude, qui est réalisée à partir d'une base de données parasitaires et pluviométriques, vise à actualiser le statut du jasside $J$. facialis en vue de mieux le prendre en compte dans la définition de nouveaux programmes de protection phytosanitaire du cotonnier en Côte d'Ivoire.

\section{MATERIEL ET METHODES Zone d'étude}

La zone d'étude, illustrée par la Figure 1, a concerné l'ensemble de la zone de culture cotonnière de la Côte d'Ivoire. Elle s'étend du Nord au Centre c'est-à-dire en région des savanes, précisément entre $6^{\circ}$ et $10^{\circ} 50$ de latitude Nord, $4^{\circ}$ et $8^{\circ}$ de longitude Ouest. Cette zone d'étude comprend l'ensemble des zones administratives des sociétés cotonnières. Au regard de la pluviométrie, ces localités sont réparties en deux grandes régions par rapport au $9^{\text {ème }}$ parallèle, à savoir : la région Nord pour toutes les localités cotonnières au-dessus du $9^{\text {ème }}$ parallèle, avec une seule saison de pluie; la région Sud pour toutes les localités cotonnières en dessous du $9^{\text {ème }}$ parallèle, avec deux saisons de pluie.

\section{Matériel}

Insecte ravageur cible : Cette étude porte sur le jasside Jacobiella facialis Jacobi, 1912 (Cicadellidae), insecte piqueur-suceur du cotonnier. C'est une cicadelle qui vit à la face inférieure des feuilles. Les larves et les adultes piquent les nervures secondaires des feuilles et introduisent une salive toxique qui provoque la décoloration du limbe. Les piqûres provoquent le jaunissement des bordures de feuilles. De fortes attaques de $J$. facialis sont susceptibles d'induire l'arrêt du développement du plant ou la chute des organes de reproduction (Selvaraj et al., 2011).

\section{Méthodes}

\section{Choix des parcelles par zone cotonnière}

Chaque année, dans les différentes zones de production cotonnière, ont été sélectionnées dix (10) parcelles paysannes par zone. Le nombre de zones a varié entre 32 et 38 localités, selon les années, pour des raisons techniques dépendantes des sociétés cotonnières. Le dispositif de suivi parasitaire a donc impliqué un total annuel de 320 à 380 parcelles paysannes. Les parcelles paysannes ont été proportionnellement réparties sur les principales décades de semis dans la région. Il s'agit pour la région Nord où les semis commencent dès la mi-mai, des décades de semis suivantes : une (1) pour la troisième décade de mai, trois (3) pour la première décade de juin, trois (3) pour la deuxième décade de juin, deux (2) pour la troisième décade de juin et une (1) pour la première décade de juillet. En ce qui concerne la région Sud, où les semis commencent en début juin, les parcelles ont été choisies : une (1) pour la première décade de juin, trois (3) pour la deuxième décade de juin, trois (3) pour la troisième décade de juin, deux (2) pour la première décade de juillet et enfin une (1) pour la deuxième décade de juillet. Chacune des parcelles d'observations mesurant $1 / 4$ 
hectare $\left(2500 \mathrm{~m}^{2}\right)$, a été délimitée. Il est admis que les planteurs ont soumis ces parcelles à l'instar de tous leurs champs au programme de traitements insecticides vulgarisé.

\section{Stratégie de protection phytosanitaire}

La stratégie de protection en vigueur est basée sur un programme calendaire de 6 traitements insecticides tous les 14 jours à partir du $45^{\text {ème }}$ jour jusqu'au $115^{\text {ème }}$ jour après levée (JAL) du cotonnier. Les traitements insecticides ont été réalisés à l'aide de produits validés par la Recherche cotonnière et homologués par le Service de la Protection des Végétaux. Il s'agit de produits binaires (généralement, des associations pyréthrinoïdes + organophosphorés / néonicotinoïdes) ou de produits simples (familles chimiques alternatives aux pyréthrinoïdes).

\section{Méthode d'échantillonnage}

Sur chaque parcelle d'observations, il a été effectué une série de 15 relevés parasitaires à partir du $30^{\text {ème }} \mathrm{JAL}$ (Jour Après Levée), à intervalles réguliers d'une semaine, jusqu'au $128^{\text {ème }}$ JAL. Les observations des dégâts sur plants ont été faites plant par plant, sur un échantillon de 30 plants pris par groupes de 5 plants consécutifs par ligne, suivant la méthode séquentielle dite "de la diagonale » (Bruno et al., 2000 ; Nibouche et al., 2002). Sur chaque plant retenu, les 5 feuilles terminales ont été examinées. Le plant est considéré attaqué lorsque l'une de ces feuilles présente des dégâts ou des symptômes d'attaques de jasside. Le comptage des plants attaqués a permis d'établir les niveaux d'infestations dues aux jassides. Les observations parasitaires ont été effectuées par les agents du Service Recherche et Développement des sociétés cotonnières, formés à cet effet. L'absence des données en 2002, 2008, 2009 et 2010 s'explique par la crise militaro-politique qu'a connu la Côte d'Ivoire.

\section{Collecte des données pluviométriques}

Les données pluviométriques ont été mises à disposition par le laboratoire central sols, eaux et plantes du Centre National de Recherche Agronomique (CNRA). Les données ont été obtenues des relevés de la pluviométrie journalière effectués de juin à août au cours des périodes 1993-2007 et 2011-2015 à partir de postes météorologiques installés non loin des parcelles paysannes. Le choix de ces mois se justifie par le fait qu'ils incluent les périodes végétatives susceptibles d'abriter les toutes premières populations de jassides sur le cotonnier.

\section{Analyses des données}

Les données parasitaires et pluviométriques obtenues ont été saisies sur Excel version 2013 pour Windows en vue de l'établissement des hauteurs moyennes mensuelles de la pluviométrie ainsi que des niveaux moyens annuels et saisonniers des infestations.

Le logiciel IBM SPSS Statistics 20 a permis de faire l'analyse de variance. En cas de différence significative (seuil de 0,05) le test de Duncan a permis de dégager les différents groupes homogènes qui sont représentés par des lettres alphabétiques. Ainsi, les moyennes suivies des mêmes lettres ne sont pas différentes significativement. Les barres d'erreurs sur les histogrammes ont été représentées à partir des valeurs de l'erreur standard rattachée à chacune de ces moyennes.

Le logiciel de cartographie Surfer version 11.5.1069 qui se prête bien à la spatialisation de données a permis d'établir les cartes de distribution géographique de la pluviométrie et des infestations parasitaires. 


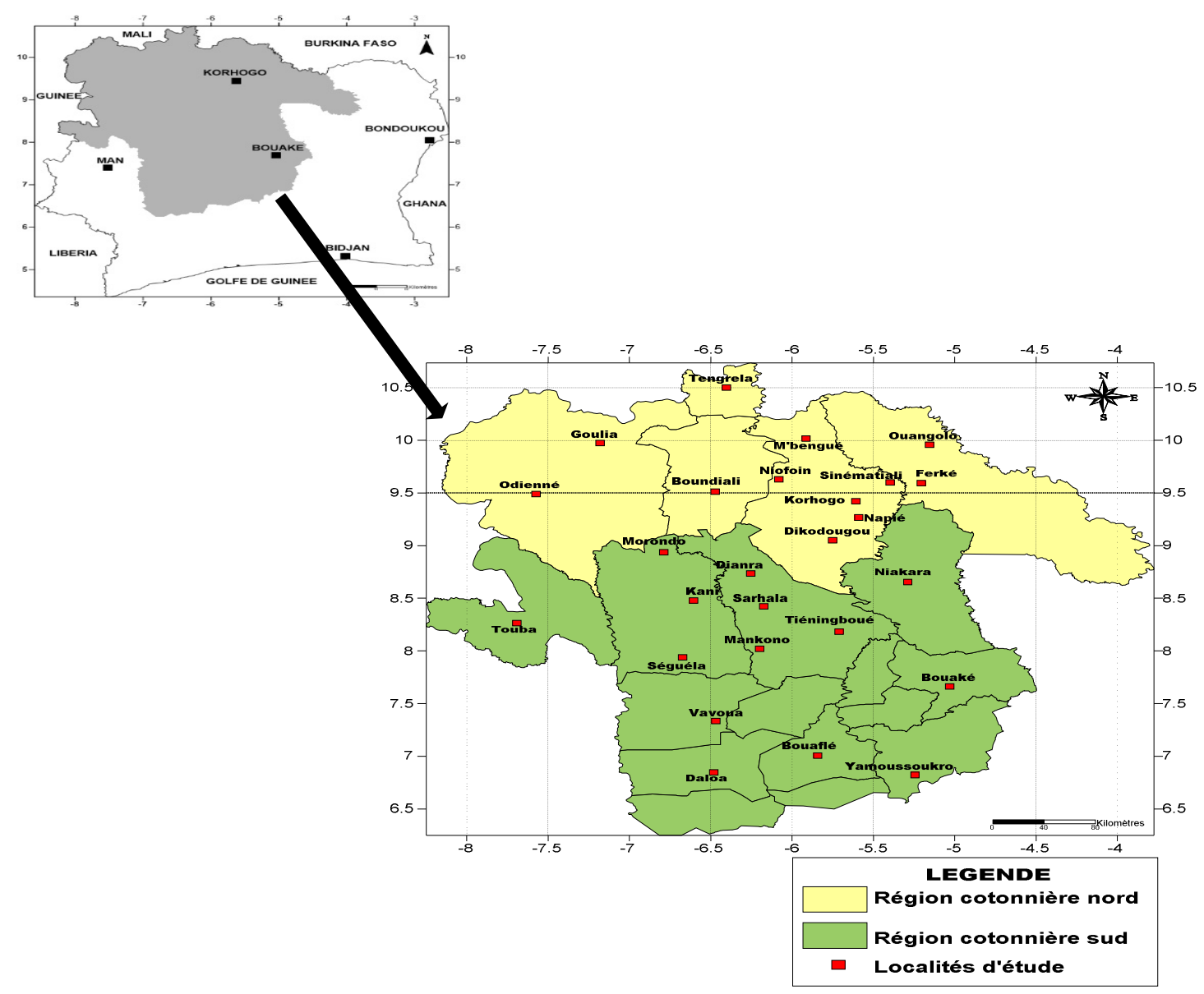

Figure 1: Zone de production cotonnière de Côte d'Ivoire.

\section{RESULTATS}

Variation annuelle des niveaux de dégâts de 1993 à 2015

Les niveaux moyens annuels des attaques du jasside $J$. facialis ont fluctué considérablement d'une année à l'autre dans la zone cotonnière de 1993 à 2015. Comme présenté par la Figure 2, les dégâts ont subi entre 1993 et 2015 une augmentation régulière de 0,52 à 6,01 plants attaqués pour 30 plants. Deux périodes se sont distinguées dans l'évolution des attaques: une première phase (1993-2007) correspondant à de faibles niveaux de dégâts, variant entre 0,52 et 2,19 plants attaqués pour 30 plants; et une seconde phase (2011-2015) correspondant à de forts niveaux d'attaques, variant entre 3,05 et 6,01 plants attaqués pour 30 plants. La moyenne générale de cette dernière période a été estimée à 3,97 plants attaqués pour 30 plants contre un niveau moyen inférieur de 1,41 plant attaqué pour 30 plants pour la période précédente.

\section{Distribution géographique des attaques}

$\mathrm{La}$ distribution géographique des dégâts du jasside $J$. facialis est illustrée par la Figure 3, présentant d'importantes modifications pour les périodes 1993-2007 et 2011-2015.

Pour la première période, les variations géographiques ont été très homogènes sur 
l'ensemble des zones de production (Figure $3 a)$, en dépit du fait qu'un certain nombre de localités ont eu une pression parasitaire audessus de celle de la moyenne générale d'infestations (1,21 plant attaqué pour 30 plants). Il s'est agit de la région Nord-Ouest de la grande zone de production, située entre les longitudes $6^{\circ}-7^{\circ}$ Ouest et les latitudes $8,5^{\circ}$ $10^{\circ}$ Nord, comprenant les localités de Niofoin (4,19 plants attaqués pour 30 plants), Sarhala (2,29 plants attaqués pour 30 plants), Dianra ( 3,01 plants attaqués pour 30 plants) et Kani ( 2,32 plants attaqués pour 30 plants).

La seconde période, contrairement à la première, a présenté une grande variabilité géographique avec de fortes infestations généralisée dans le Nord-Est des zones de production cotonnière (Figure $3 b$ ). De nombreuses localités ont eu une pression parasitaire au-dessus de 4 plants attaqués pour 30 plants. Elles sont généralement implantées dans le Nord-Est de la grande zone de production, située entre les longitudes $5^{\circ}-6,5^{\circ}$ Ouest et les latitudes $9^{\circ}-10,5^{\circ}$ Nord, comprenant les localités de Sinématiali $(15,53$ plants attaqués pour 30 plants) et Ferké $(9,19$ plants attaqués pour 30 plants). Les niveaux de dégâts ont été particulièrement plus accrus dans les localités situées le long de la longitude $5,5^{\circ}$ Ouest.

\section{Variation saisonnière des niveaux de dégâts}

L'évolution saisonnière des dégâts comme l'illustre la Figure 4, montre la présence du ravageur $J$. facialis durant tout le cycle cultural du cotonnier, avec cependant une variation dans le profil selon qu'il a été établi pendant les périodes 1993-1994 et 2012-2015.

Pour la première période, correspondant à une période de moindre importance des dégâts de J. facialis, les fortes infestations du jasside étaient observées en début de cycle du cotonnier, entre les $30^{\text {ème }}$ et $44^{\text {ème }}$ JAL. Après le pic de pullulations, les niveaux d'infestations tendent à décroître pour totalement s'annuler vers la fin du cycle cultural du cotonnier (Figure 4a). Contrairement à la première période, en 2012-
2015 correspondant à une période où les dégâts de $J$. facialis le classent parmi les ravageurs émergents, les infestations ont été enregistrées sur tout le cycle cultural du cotonnier et ont été en constante hausse du début à la fin du cycle du cotonnier (Figure $4 b)$. Les plus fortes infestations ont été notées entre les $93^{\text {ème }}$ et $114^{\text {ème }}$ JAL selon les années $\left(5,76\right.$ plants attaqués pour 30 plants au $93^{\text {ème }}$ JAL en $2012 ; 8,24$ plants attaqués pour 30 plants au $114^{\text {eme }}$ JAL en $2013 ; 8,84$ plants attaqués pour 30 plants au $107^{\text {z̀me }}$ JAL en 2014 et 9,86 plants attaqués pour 30 plants au $93^{\text {ème }}$ JAL en 2015).

Incidence des décades de semis sur l'abondance saisonnière de Jacobiella facialis

Le nombre moyen de plants attaqués par J. facialis, au cours des périodes d'observations, a varié en fonction des dates de semis comme présenté par la Figure 5. De façon générale, les niveaux de dégâts sur les cotonniers de toutes les décades de semis de 2015 ont été supérieurs à ceux des décades de 1993-1994. L'analyse statistique révèle une différence significative entre les moyennes de plants attaqués sur les deux périodes. En 1993-1994, les faibles infestations ont été obtenues aux décades D1, D2, D3 et D6 (respectivement 2,$01 ; 2,18 ; 1,77$ et 1,56 plants attaqués pour 30 plants). En 2015, les plus faibles infestations ont été obtenues uniquement à la D6 (3,02 plants attaqués pour 30 plants).

\section{Distribution de la pluviométrie}

Pendant la période 1993-2007, la distribution géographique de la pluviométrie de juin-juillet $a$ été très homogène sur l'ensemble des zones de production (Figure 6a). Les niveaux de pluie enregistrés dans les localités de la zone cotonnière, s'étendant d'Odienné à Ferké, entre les longitudes $5^{\circ}-8^{\circ}$ Ouest ont été supérieurs à $320 \mathrm{~mm}(396,17$ $\mathrm{mm}$ à Odienné contre $326,80 \mathrm{~mm}$ à Ferké). Contrairement à la première, la distribution géographique de la pluviométrie pendant la période 2011-2015 (Figure 6b) a montré une 
forte régression de la pluviométrie dans l'ensemble des zones de production, avec des niveaux particulièrement inférieurs à $320 \mathrm{~mm}$ dans les zones Nord-Est, s'étalant de Boundiali à Ferké, comprises entre les longitudes $5^{\circ}-6,5^{\circ}$ Ouest et les latitudes $9^{\circ}$ $10,5^{\circ}$ Nord.

L'analyse de la corrélation entre les niveaux de dégâts du jasside $J$. facialis et la pluviométrie saisonnière pendant les années 1993-2015, comme présenté par le Tableau 1, montre que dans la plupart des régions, la corrélation est négative. Cela est plus marqué dans la région de Korhogo avec des valeurs de corrélations significatives $(\mathrm{p}<0,05)$ en juillet et juin-juillet.

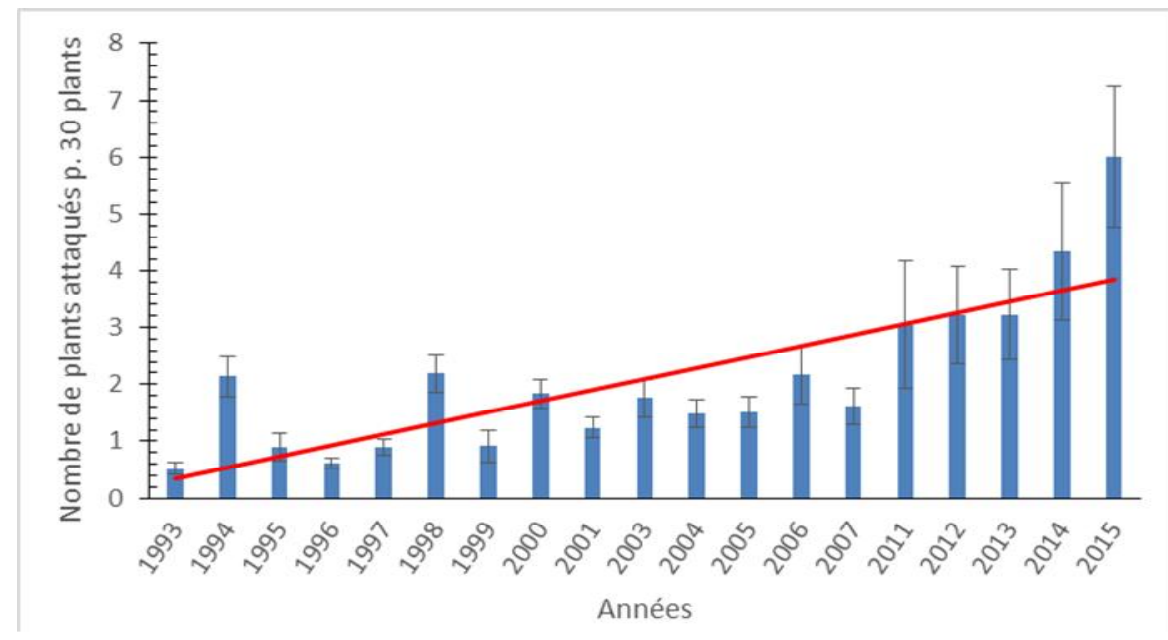

Figure 2: Evolution du niveau annuel de plants attaqués par Jacobiella facialis.
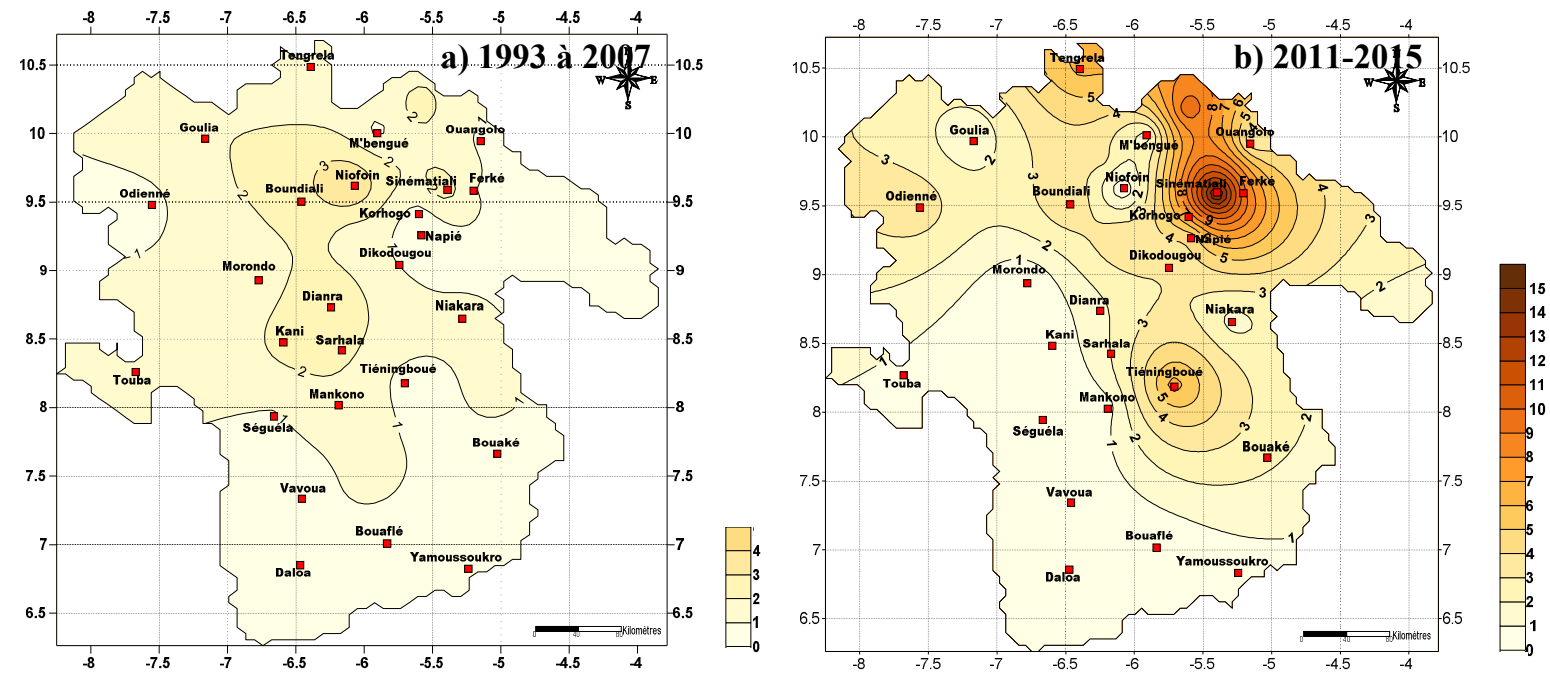

Figure 3: Distribution géographique des niveaux d'infestations de Jacobiella facialis en zone de production cotonnière. 

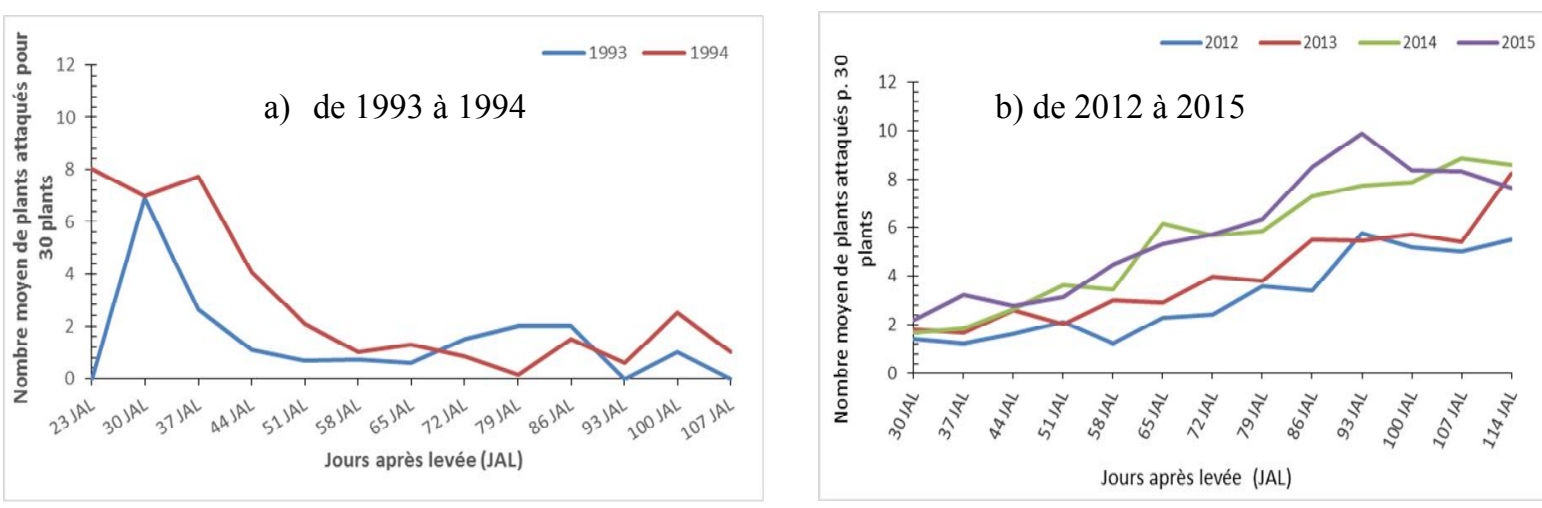

Figure 4 : Evolution saisonnière du nombre de plants attaqués par Jacobiella facialis.
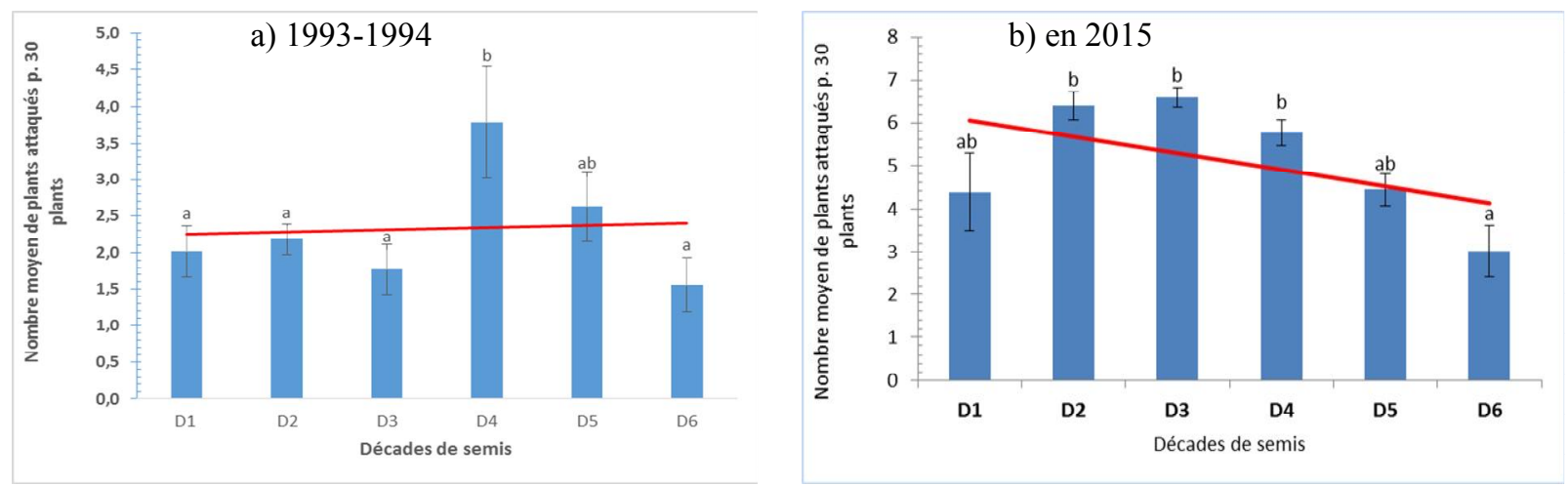

Figure 5: Abondance du nombre de plants attaqués par Jacobiella facialis en fonction des décades de semis.
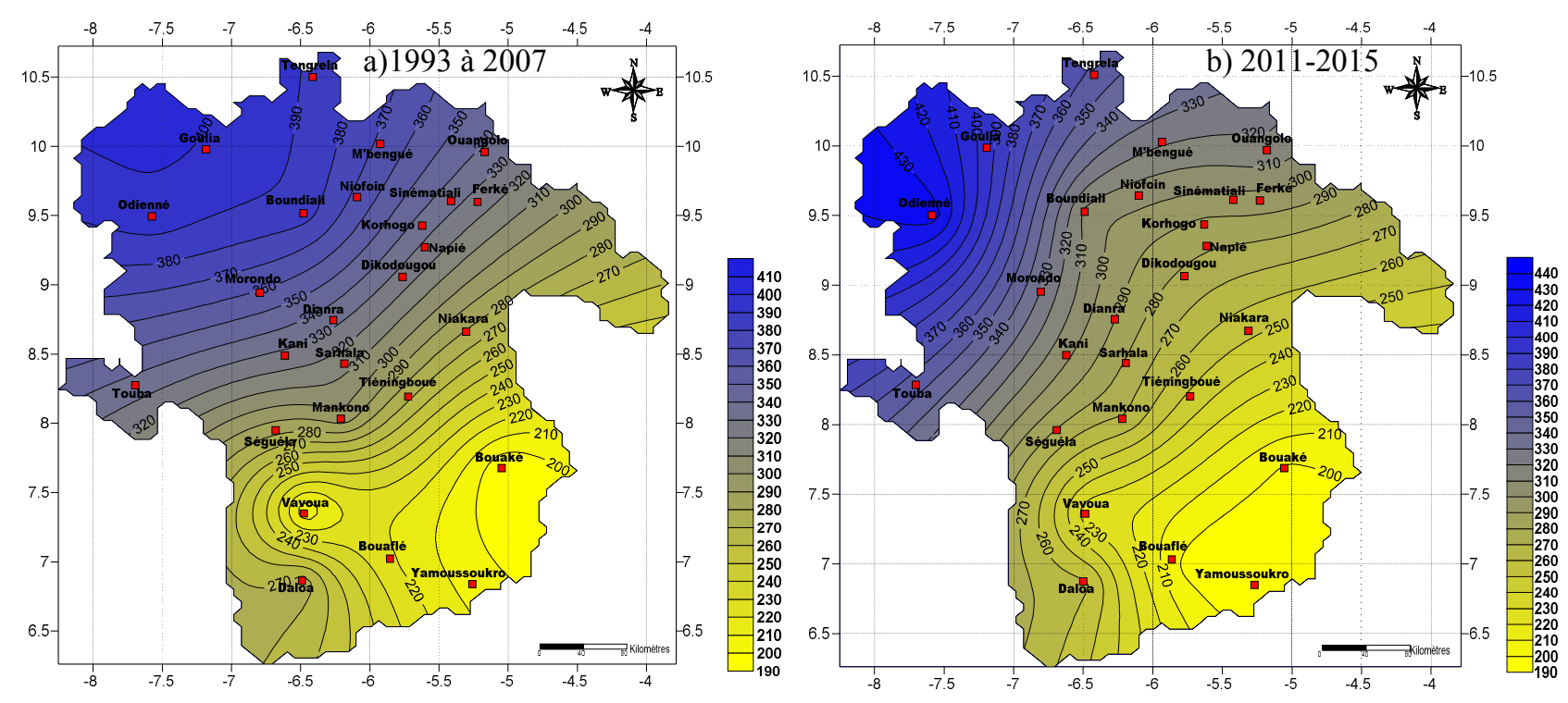

Figure 6: Distribution de la pluviométrie en zone de production cotonnière de juin-juillet. 
Tableau 1: Corrélation entre les niveaux de dégâts du jasside J. facialis et la pluviométrie saisonnière pendant les années 1993-2015.

\begin{tabular}{crrrcccc}
\hline \multirow{2}{*}{ Bouaflé } & & Juin & Juil. & Août & Juin-Juil. & Juil.-Août & Juin-Juil.-Août \\
& $\mathrm{r}$ & $-0,089$ & $-0,128$ & $-0,273$ & $-0,153$ & $-0,267$ & $-0,305$ \\
& Sign. & 0,771 & 0,677 & 0,367 & 0,617 & 0,377 & 0,311 \\
\hline \multirow{2}{*}{ Niakara } & $\mathrm{r}$ & $-0,050$ & $-0,542$ & 0,159 & $-0,375$ & $-0,204$ & $-0,165$ \\
& Sign. & 0,906 & 0,165 & 0,707 & 0,361 & 0,628 & 0,697 \\
\hline \multirow{2}{*}{ Korhogo } & $\mathrm{r}$ & $-0,118$ & $-0,603$ & 0,037 & $-0,572$ & $-0,329$ & $-0,322$ \\
& Sign. & 0,674 & $0,017^{*}$ & 0,896 & $0,026^{*}$ & 0,231 & 0,243 \\
\hline \multirow{2}{*}{ Mankono } & $\mathrm{r}$ & $-0,483$ & 0,152 & $-0,017$ & $-0,286$ & 0,091 & $-0,233$ \\
& Sign. & 0,080 & 0,604 & 0,955 & 0,321 & 0,757 & 0,423 \\
\hline \multirow{2}{*}{ Ouangolo } & $\mathrm{r}$ & 0,301 & $-0,241$ & $-0,009$ & $-0,078$ & $-0,154$ & $-0,056$ \\
& Sign. & 0,295 & 0,406 & 0,976 & 0,790 & 0,600 & 0,849 \\
\hline \multirow{2}{*}{ Vavoua } & $\mathrm{r}$ & $-0,050$ & $-0,251$ & $-0,462$ & $-0,184$ & $-0,419$ & $-0,402$ \\
& Sign. & 0,877 & 0,432 & 0,131 & 0,567 & 0,175 & 0,196 \\
\hline \multirow{2}{*}{ Odienné } & $\mathrm{r}$ & 0,495 & $-0,104$ & 0,185 & 0,311 & 0,099 & 0,275 \\
& Sign. & 0,146 & 0,776 & 0,609 & 0,382 & 0,786 & 0,443 \\
\hline \multicolumn{2}{r}{$\mathrm{r}=$ coefficient de corrélation } & \multicolumn{2}{c}{ Sign $=$ significativité à 0,05} & &
\end{tabular}

\section{DISCUSSION}

Les résultats de cette étude ont mis en évidence un changement dans le statut parasitaire du jasside qui se traduit par une augmentation au fil des années du nombre de dégâts et un déplacement de la zone géographique de prédilection du ravageur. Les données pluviométriques ont fait ressortir une corrélation négative entre la pluviométrie et les variations des niveaux de dégâts causés par J. facialis.

La pluviométrie est l'un des facteurs environnementaux qui influencent la prolifération des jassides en culture cotonnière (Selvaraj et al., 2011; Asifa et al., 2014). Selon Chandani et Sathe (2015), les perturbations climatiques, caractérisées par des pluies à faible intensité et de faibles valeurs d'humidité relative, seraient favorables au développement de cet insecte. La distribution parasitaire observée actuellement pourrait être due à une réduction de la zone géographique à forte intensité pluviométrique et cela, surtout pendant la phase végétative du cotonnier, stade de croissance qui est pourtant favorable à l'apparition et la prolifération des populations de jassides connus pour être des ravageurs apparaissant très tôt au cours du cycle biologique de la plante.

La recrudescence du ravageur peut être aussi la conséquence de plusieurs autres facteurs agro-écologiques. Le recours presqu'exclusif aux produits chimiques par les producteurs pour lutter contre les ravageurs pourrait entraîner une modification de l'entomofaune à travers l'élimination des ennemis naturels de certains ravageurs (Imam et al., 2010 ; Gopal et Tarikul, 2014). De plus, il est à signaler, par ailleurs, que pendant des décennies, les stratégies de protection ont essentiellement été dirigées contre les chenilles de la capsule qui sont les principales responsables des pertes de récoltes (Martin et al., 2000 ; Ochou et al., 2012). Cette situation pourrait avoir contribué à l'émergence 
actuelle de certains ravageurs autrefois sans intérêt majeur, comme J. facialis. La persistance des dégâts de jasside, malgré les applications insecticides, pourrait également traduire une perte de sensibilité vis-à-vis des matières actives utilisées, comme cela a été le cas de Helicoverpa armigera vis-à-vis des pyréthrinoïdes en Côte d'Ivoire depuis 1998 (Martin et al., 2000). En effet, les matières actives telles que le profénofos et l'acétamipride sont largement utilisées respectivement depuis plus d'une quarantaine et une quinzaine d'années contre ce ravageur dans les programmes de protection en vigueur dans le pays. Or il a été montré, ailleurs, que ce ravageur est capable de développer de la résistance aux insecticides (Sagar et al., 2013 ; Sagar et Balikai, 2015; Halappa et Patil, 2016). Il serait donc opportun de déterminer le statut de la sensibilité aux insecticides utilisés chez ce ravageur afin de rechercher éventuellement des molécules alternatives. Par ailleurs, en Côte d'Ivoire, la lutte contre les jassides était également basée en grande partie sur la sélection de variétés à forte pilosité foliaire (Bruno et al., 2000). Ce caractère confère à la plante un bon degré de tolérance à ce ravageur (Eittipibool et al., 2001). Au vu des pullulations du jasside $J$. facialis ces dernières années, il conviendrait d'analyser, avec attention, le degré de pilosité des nouvelles variétés vulgarisées.

$\mathrm{Au}$ cours des dernières années, la présence de $J$. facialis a été notée tout au long $\mathrm{du}$ cycle biologique du cotonnier avec une augmentation au fil du temps du nombre moyen de plants attaqués. Cet état de fait traduit, d'une part, une faiblesse $d u$ programme de protection actuellement en vigueur pour le contrôle du ravageur. D'autre part, la modification du cycle de l'insecte pourrait être favorisée par une baisse des pluies et une mauvaise répartition de celles-ci dans le temps, créant ainsi des conditions adéquates au développement des jassides.

Les variations révélées entre les décades de semis permettent de noter une influence de la date de semis sur les niveaux d'attaques de jassides. L'influence des dates de semis sur les populations de ravageurs a elle été mise en évidence par certains auteurs (Hala et al., 2006 ; Hermann et al., 2015). En effet, la présence de certains ravageurs est liée en partie à la phénologie de la plante. D'autres sont beaucoup plus dépendants des facteurs saisonniers. Il existe une forte pression parasitaire des jassides sur l'ensemble des décades de semis de 2015 par rapport à la période antérieure.

\section{Conclusion}

La présente étude a mis en évidence, au cours de ces dernières années, l'ampleur des dégâts du jasside $J$. facialis et sa présence quasi-permanente durant tout le cycle du cotonnier, ainsi qu'un changement considérable dans l'aire de distribution des attaques. Ces changements dans le statut du ravageur, qui pourraient s'expliquer par une modification des conditions agro-écologiques (perturbations climatiques, produits à faible activité cicadellicide, résistance du ravageur aux insecticides utilisés, faible pilosité des variétés, disparition des ennemis naturels, etc.) sont susceptibles de limiter l'efficacité des programmes de protection. Cette situation parasitaire émergente demande une révision du programme de protection en vigueur, afin de mieux prendre en compte le nouveau faciès parasitaire $\mathrm{du}$ ravageur. Pour renforcer l'efficacité biologique du programme de protection, il est indispensable de traiter les semences avec des produits systémiques ou d'utiliser les molécules alternatives efficaces pour les traitements foliaires. Il s'avère aussi nécessaire de rechercher des méthodes alternatives en vue de réduire le recours aux 
produits chimiques et de prévenir des cas de résistance.

\section{CONFLIT D'INTERETS}

Les auteurs déclarent n'avoir aucun conflit d'intérêts concernant cet article.

\section{CONTRIBUTIONS DES AUTEURS}

PWEK, initiatrice de l'article, a saisi les données parasitaires et pluviométriques, a procédé à l'analyse des données, à leur interprétation et à la rédaction du document. GECO et GJRD ont contribué à la révision des données saisies et analysées, et à la révision critique du contenu de l'article. SCD a contribué à l'analyse des données pluviométriques et à l'élaboration des cartes de distribution géographique des niveaux de dégâts et de pluviométrie. MK et KKNB ont contribué à la formation des agents des services Recherche et Développement des sociétés cotonnières qui ont assuré les observations parasitaires sur le terrain, et à la révision critique du contenu de l'article. DM a contribué à l'interprétation des données et à la révision critique du contenu de l'article. OGO initiateur de l'activité de recherche, a élaboré la méthodologie mise en œuvre, mis à disposition les données, a contribué à la formation des agents des services Recherche et Développement des sociétés cotonnières ainsi qu'à l'analyse des données et leur interprétation, a orienté, lu et corrigé le document.

\section{REFERENCES}

Asifa H, Muhammad SS, Abid M, Saghir A, Noor UI. 2014. Forecasting and modeling of sucking insect complex of cotton under agro-ecosystem of MultanPunjab, Pakistan. J. Agri. Sci., 51(4): 997-1003. http://pakjas.com.pk/papers/ 2369.pdf
Badiane D, Gueye MT, Coly EV, Faye O. 2015. Gestion intégrée des principaux ravageurs du cotonnier au Sénégal et en Afrique occidentale. Int. J. Biol. Chem. Sci., 9(5): 2654-2667. DOI : http://dx.doi.org/10.4314/ijbcs.v9i5.36

Bruno M, Mamoutou T, Idrissa T, Navigué NT. 2000. La lutte contre les ravageurs du cotonnier au Mali : problématique et évolution récente. Cahiers Agricultures, $9: 109-115$.

Chandani SK, Sathe TV. 2015. Incidence and host plants for Amrasca biguttula (Ishida) from Kolhapur Region, India. International Journal of Development Research, 5(3): 3658-3661. Available online at http://www.journalijdr.com

Djihinto CA, Affokpon A, Dannon E, Bonni G. 2016. Le profenofos, un alternatif à l'endosulfan en culture cotonnière au Bénin. Int. J. Biol. Chem. Sci., 10(1): 175-183. DOI: http://dx.doi.org/ 10.4314/ijbcs.v10i1.12

Eittipibool W, Renou A, Chongrattanameteekul W, Hormchan P. 2001. Effects of Cotton Growth Regulator on Jassid Infestation and Injury. Kasetsart J. Nat. Sci., 35: 378385.

Gopal D, Tarikul I. 2014. Relative efficacy of some newer insecticides on the mortality of jassid and white fly in brinjal. International Journal of Research in Biological Sciences, 4(3): 89-93.

Hala N, Ochou GO, Foua Bi K, Allou K, Ouraga Y, Kouassi P. 2006. Dynamique spatio-temporelle des populations d'altises, Podagrica spp (Coleoptera: Chrysomelidae): implications agronomiques en zones cotonnières de Côte d'Ivoire. Agronomie Africaine, 18(1): 41-57. http://dx.doi.org/ 10.4314/aga.v18i1. 1678 
Halappa B, Patil RK. 2016. Detoxifying enzyme studies on cotton leafhopper, Amrasca biguttula biguttula (Ishida), resistance to neonicotinoid insecticides in field populations in Karnataka, India. Journal of Plant Protection Research, 56(4): 346-352. DOI: https://doi.org/ 10.1515/jppr-2016-0047

Herman MB, Moumouni I, Mere SBJTO. 2015. Contribution à l'amélioration des pratiques paysannes de production durable de coton (Gossypium hirsutum) au Bénin: cas de la commune de Banikoara. Int. J. Biol. Chem. Sci., 9(5): 2401-2413. DOI : http://dx.doi.org/ 10.4314/ijbcs.v9i5.12

Imam TS, Yusuf AU, Mukhtar MD. 2010. A survey of some insect pests of cultivated vegetables in three selected irrigation areas along Jakara river, Kano, Nigeria. Int. J. Biol. Chem. Sci., 4(2): 400-406. DOI: $10.4314 /$ ijbcs.v4i2.58136

Martin T, Ochou OG, Hala NF, Vassal JM, Vaissayre M. 2000. Pyrethroid resistance in the cotton bollworm, Helicoverpa armigera (Hübner), in West Africa. Pest. Manag. Sci., 56: 549-554. DOI: $10.1002 /($ SICI)1526-4998 (200006)56:6<549::AID-PS160>3.0.CO; $2-\mathrm{Y}$

Nibouche S, Beyo J, Goze E. 2002. Mise au point de plans d'échantillonnage pour la protection sur seuil contre les chenilles de la capsule du cotonnier. Actes du colloque, 27-31 mai 2002, Garoua, Cameroun, p. 6.

Ochou OG, Doffou NM, N'goran KE, Kouassi KP. 2012. Impact de la gestion de la résistance aux pyréthrinoïdes sur l'évolution spatio-temporelle des principaux lépidoptères carpophages du cotonnier en Côte d'Ivoire. J. Appl. Biosci., 53: 3831-3847.

Ochou OG, Martin T. 2002. Pyrethroïd Resistance in Helicoverpa armigera (Hübner): Recent Developments and Prospects for its Management in Côte d'Ivoire West Africa. Resist. Pest. Manag., 12(1):10-16.

Sagar D, Balikai RA, Khadi BM. 2013. Insecticide resistance in leafhopper, Amrasca Biguttula biguttula (Ishida) of major cotton growing districts of Karnataka, India. Biochem. Cell. Arch., 13(2): 261-265.

Sagar D, Balikai RA. 2015. Toxicity of neonicotinoids against cotton leafhopper, Amrasca biguttula biguttula (Ishida) in Karnataka, India. Res. on Crops, 16(1): 170-175. DOI: $10.5958 / 2348$ 7542.2015.00024.8

Sarr M, Badiane D, Sane B. 2016. Evaluation de l'efficacité de nouveaux programmes de protection phytosanitaire contre les principaux ravageurs du cotonnier Gossypium hirsutum L. au Sénégal. Int. J. Biol. Chem. Sci., 10(5): 2163-2174. DOI: ttp://dx.doi.org/10.4314/ijbcs.v10i5.18

Selvaraj S, Adiroubane D, Ramesh V. 2011. Population dynamics of leafhopper, Amrasca devastans Distant in cotton and its relationship with weather parameters. Journal of Entomology, 8(5): 476-483. DOI:http://dx.doi.org/10.3923/je.2011.4 76.483 . 